\title{
An Automatic Technique for Power Line Pylon Detection from Point Cloud Data
}

\author{
Mohammad Awrangjeb*, David Jonas ${ }^{\dagger}$ and Jun Zhou* \\ *Institute for Integrated and Intelligent Systems \\ Griffith University, Nathan, QLD 4111, Australia \\ Email: m.awrangjeb@griffith.edu.au,jun.zhou@griffith.edu.au \\ ${ }^{\dagger}$ AAM Pty Limited, Brisbane, QLD 4000, Australia \\ E-mail: d.jonas@aamgroup.com
}

\begin{abstract}
This paper proposes a new pylon detection technique from point cloud data. Two masks are created from the non-ground points that mainly represent trees and power line components. The first mask is the power line mask $M_{m}$, which contains the power line components and trees and where successive pylons are found connected with wires. The second mask is the pylon mask $M_{p}$, where successive pylons are found disconnected, and thus is exploited to obtain candidate pylons using a connected component analysis. By contrasting the area, shape and symmetry properties between trees and pylons majority of the false candidates (trees) are removed from $M_{p}$. Finally, long straight lines that represent wires between successive pylons are extracted from $M_{m}$ and used to remove the remaining trees from $M_{p}$. Experimental results show that the proposed technique provides a high pylon detection rate in terms of both completeness (100\%) and correctness (100\%).
\end{abstract}

\section{INTRODUCTION}

Reliable mapping of electrical power line corridors (PLCs) is important for a number of applications, such as monitoring of vegetation encroachment within a PLC [1] and analysis of power line structural stability [2]. In fact, adding spatial context to asset (e.g., power line) management significantly improves the efficiency of managing an asset which involves millions of components, spread across thousands of linear kilometres and impacting diverse land uses. Traditionally, PLCs are surveyed in person or by manually inspecting aerial photos and videos. However, periodic manual inspection of thousands of kilometres of PLCs is not only time consuming and labour intensive, but also subject to errors due to involvement of human judgement. These inspection technologies are all spatially based, so a reliable map base is essential to gain the maximum benefit. Furthermore, the map of most utilities may be out of date due to inaccuracies of original surveys and alterations to the network. Therefore, utility companies are seeking the benefits of modern technology to monitor the condition of their assets and reduce maintenance costs. Fortunately, the advent of airborne laser scanning technology, also known as LIght Detection And Ranging (LIDAR), has made the survey automatic and economic. By using the LIDAR technology, millions of 3D points on the earth surface and objects on it are collected and then processed off-line on powerful computers automatically. Thus, the LIDAR technology has made the entire survey more effective and efficient. A recent comprehensive review of various types of PLC surveying methods can be found in Matikainen et al. [3].

The processing of LIDAR data for 3D mapping of PLC has two main steps [4]: Points are first classified or segmented into different objects such as trees, pylons, wires and ground. Then, points on individual power line span are exploited to model wires between successive pylons. However, a well modelling of thin power lines requires a high density input data. Thus, there can be millions of points in a given $1 \mathrm{~km}^{2}$ scene. However, the actual number of points reflected from wires and pylons is far smaller than the number of input points. The detection of pylons can make the wire extraction step faster as in this case only the non-ground points between successive pylons need to be processed. Most existing power line extraction methods (e.g., McLaughlin [4]) overlook the detection of pylons as a pre-requisite for power line extraction, except Sohn et al. [5]. Nonetheless, the absence of pylons can make a 3D PLC map incomplete and less useful.

In the literature, pylons are usually classified as a separate class using supervised classifiers, e.g., Random Forest (RF) [6] and JointBoost [2]. The classification output consists of a set of points for each class. Thus, 3D positions of individual pylons are not provided. Moreover, supervised classifiers have two main requirements: large training data set and balanced learning [7]. A large training data set for pylons is in general hard to get for a given test scene. For example, the data set used by Kim and Sohn [6] had only $0.81 \%$ points for pylons. So, pylons are in general a minority class compared to trees, buildings and wires in any input data. Using such an unbalanced classes of data, supervised classifiers tend to learn incorrect classification toward minority classes [6].

Sohn et al. [5] proposed the only pylon detection technique that provided individual pylon locations. 3D lines were first extracted using RANSAC (RANdom SAmple Consensus) algorithm. Then, these lines were converted into a binary image, from which $2 \mathrm{D}$ lines were classified using the RF classifier based on the orientation and parallelism properties. Finally, non-pylon objects were removed using a voting scheme based on contextual relations among the lines.

This paper proposes a new and classifier-free pylon detection technique from point cloud data. The proposed technique is based on the rules derived from the intuitive observation 


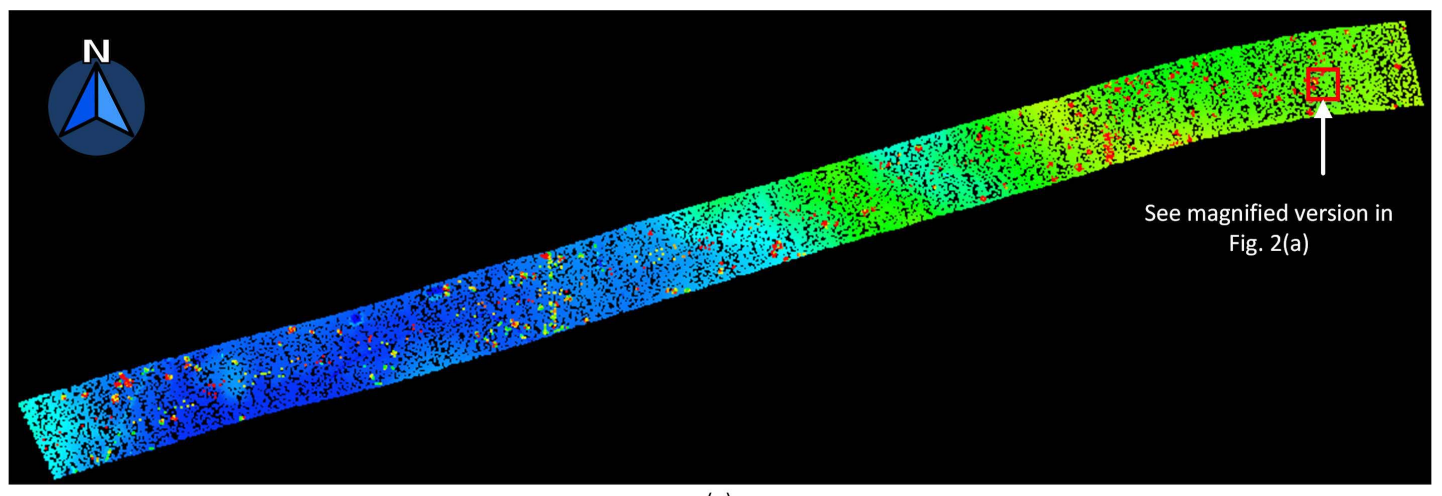

(a)

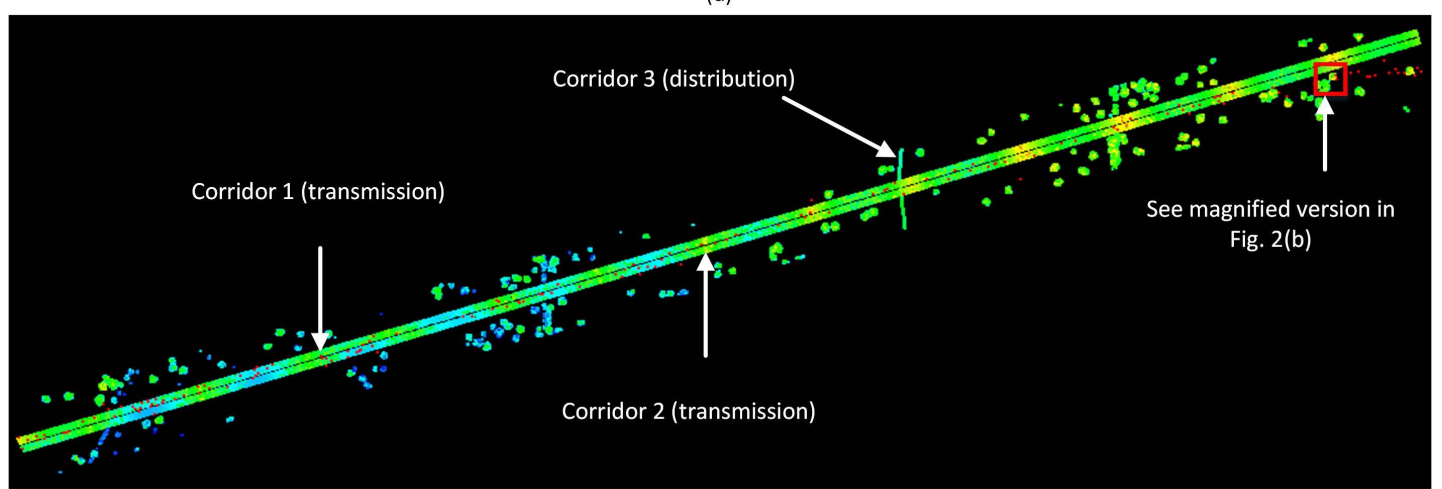

(b)

Fig. 1: Test data set $(5,560 \mathrm{~m} \times 330 \mathrm{~m})$ : (a) input point cloud data and (b) non-ground points. ' $\mathrm{N}$ ': north direction; Point colours: relative heights of points: 'red' = high, 'green' = medium, 'blue' = low.

about the geometric property of objects in the scene. For instance, while pylons have a symmetric and/or cylindrical shape, trees mostly possess a random shape mainly due to branches and leaves. The height histogram used to analyse the symmetry property is capable not only to remove many of the trees, but also to detect pylons with only 8 to 10 points reflected from each pylon. The long straight lines representing wires between successive pylons help in elimination of the remaining trees. Since, the proposed method does not use any supervised classifiers, it avoids the aforementioned problems associated with the classifier-based techniques.

There are two approaches to process the point cloud data: grid-based and point-based [6]. In grid-based approach 3D points are rasterised into $2 \mathrm{D}$ height image, which makes the classification and segmentation algorithms faster. However, the accuracy of the result is limited to the resolution of the image. In contrast, in point-based approach each input point is classified into one of the object classes. While the classification result is highly accurate in this approach, it is computationally expensive for a large input data.

This paper presents an integrated approach that combines the benefit of both point-based and grid-based approaches for automatic detection of pylons from point cloud data. In one hand, it generates binary masks in order to make the point processing faster by considering only those points that are within a given area of interest. On the other hand, it processes the points within each candidate pylon locations in order to capture and exploit the 3D symmetric property of pylons.

\section{Data SeT}

Figure 1 shows the test data set from Maindample, Victoria, Australia.The test site is $5,560 \mathrm{~m}$ in length and $330 \mathrm{~m}$ in width. There are 3 power line corridors ( 2 transmissions and 1 distribution). The two transmission corridors include 26 pylons (13 on each), which are columns made of steel frames. The distribution corridor has only 2 poles, which are cylindrical columns. As can be seen in Figure 2, usually the height, length and width of a pylon on a transmission line are higher (30 $\mathrm{m}, 18 \mathrm{~m}$ and $2.5 \mathrm{~m}$, respectively) than those of a pole on a distribution line $(15 \mathrm{~m}, 0.30 \mathrm{~m}$ and $0.30 \mathrm{~m}$, respectively). The width of the bunch of the power lines between two pylons is $20 \mathrm{~m}$ for transmission line and $5 \mathrm{~m}$ for distribution line. Note that unless specifically mentioned, the word 'pylon' is used for both 'pylon' and 'pole' in the rest of the paper.

The total number of points in the point cloud is $32,708,377$ (see Figure 1(a)). However, the number of non-ground points, shown in Figure 1(b), is 2,097,265 (16.5\% of the total points). Also, the number of non-ground points within the three corridors is only 279,867 ( $6.4 \%$ of the total points). Thus, the aim of the research presented in this paper is to automatically obtain all the pylons and, therefore, to effectively define the power line corridors to use in any future investigations. 


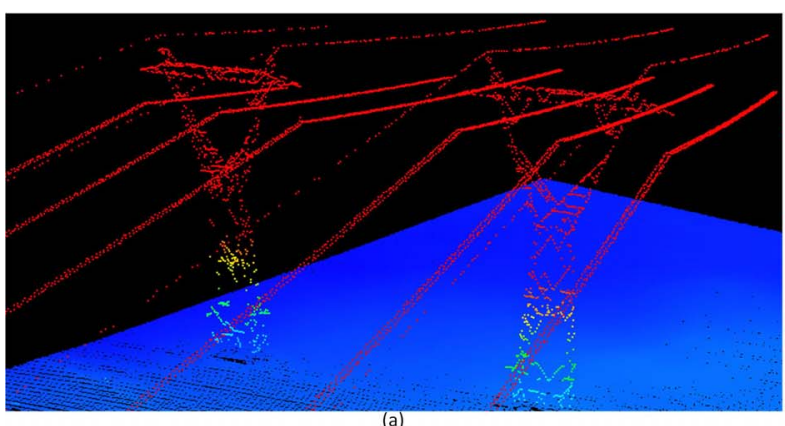

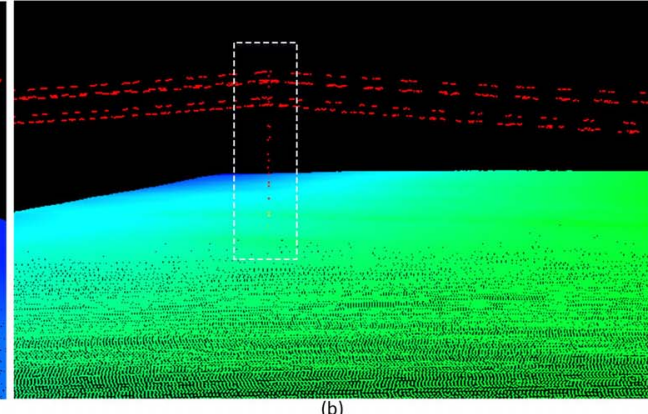

Fig. 2: Sample pylons from Figure 1: (a) Two transmission pylons and (b) one distribution pole (within white dashed rectangle). 'F': flight direction; Point colours: relative heights of points: 'red' = high, 'green' = medium, 'blue' = low.

Figure 3(a) shows a small sample scene from the test data set and it is used to describe the proposed pylon detection technique in detail below.

\section{Proposed Method}

Figure 4 shows the flowchart of the proposed pylon detection technique. The input data consists of a LIDAR point cloud and DTM (Digital Terrain Model). The DTM is a height representation of the earth surface without any objects on it.

At first, the LIDAR points are classified into two groups: ground points, such as ground, road furniture and bushes that are below a height threshold, and non-ground points, which represent elevated objects (such as power lines, pylons and trees) above this threshold. The power line mask $M_{m}$, is generated using the non-ground points. Individual pylons, power lines and trees are obtained as clusters of black pixels in $M_{m}$. Pylons and power lines of the same power line corridor are found connected in $M_{m}$, but if two or more corridors intersect (horizontally and/or vertically), then they are also found connected.

In $M_{m}$ wires and pylons are found connected and it is hard to separate them. So, the non-ground points are again processed to generate the pylon mask $M_{p}$, where pylons are found stand-alone and not connected at all with wires. The candidate pylons are obtained using a connected component analysis in the mask, followed by a removal of trees by comparing area, shape and symmetry properties of trees and pylons. Finally, the parallelism property of wires with the line connecting pair of candidate pylons is exploited to remove trees that have the same area and shape properties as pylons.

\section{A. Mask Generation}

If a DTM is not available, one can be generated from the LIDAR point cloud data using a commercial software. We assume that the DTM is given as an input to the proposed technique. Then, for each LIDAR point, the corresponding DTM height is used as the ground height, $H_{g}$. A height threshold

$$
T_{h}=H_{g}+h_{c},
$$

where $h_{c}$ is a height constant that separates low height objects from high height objects, is then applied to the LIDAR data.
Thus, the point cloud is divided into two groups: ground and non-ground points. The second group consists of the points that represent elevated objects, such as pylons, power lines and trees with heights above $T_{h}$. Many authors have used $h_{c}=2$ $\mathrm{m}$ [6] or $4 \mathrm{~m} \mathrm{[7].} \mathrm{However,} \mathrm{as} \mathrm{we} \mathrm{are} \mathrm{focusing} \mathrm{on} \mathrm{the} \mathrm{detection}$ of individual pylons, which are sometimes only 15 to $30 \mathrm{~m}$ in height, $h_{c}$ has been set at $1 \mathrm{~m}$ in this study to get most of the pylon points. Figure 3(b) shows the non-ground points obtained from the input sample data shown in Figure 3(a).

The power line (binary) mask, $M_{m}$, as shown in Figure 5(b), is generated as follows. Let the resolution of $M_{m}$ be $R_{m}$ and all pixels are initially assigned 1 (white). Then, for each nonground point the corresponding pixel in the mask is marked 0 (black). The value of $R_{m}$ is crucial here, because the input point cloud data can be sparse and/or there may not be enough points reflected from narrow wires to make the pylons on the same power line corridor connected. Ideally, in the mask there should be at least one wire between two consecutive pylons to make the whole corridor connected. However, the value of $R_{m}$ should not be large that will make the mask small in size and, thus, individual objects on the power line become too tiny and confusing in the mask. Consequently, the value of $R_{m}$ is kept fixed at $0.25 \mathrm{~m}$, but for each non-ground point a neighbourhood $\Omega=N \times N$, where

$$
N=2 n+1,
$$

is filled with 0 (black). The value of $n$ is estimated as follows:

$$
n=\left|\frac{R_{w}}{R_{m}}\right|-1,
$$

where |.| indicates the round operation and $R_{w}$ is defined as

$$
R_{w}=\left\{\begin{array}{ll}
\frac{d_{m}}{2} & \text { if } d_{m}>1.0 \\
0.5 & \text { otherwise }
\end{array},\right.
$$

where $d_{m}$ is the maximum point-to-point distance on a wire. When there are two or more wires between the pylons in a corridor, it is fine to consider the smallest value of $d_{m}$ among the wires. The minimum value of $d_{m}$ is set at 0.5 in Equation 4. Therefore, $n \geq 1$, which ensures any pair of successive pylons in a corridor are connected. Figure 5(a) shows the mask with only the non-ground point locations marked 0. As shown 


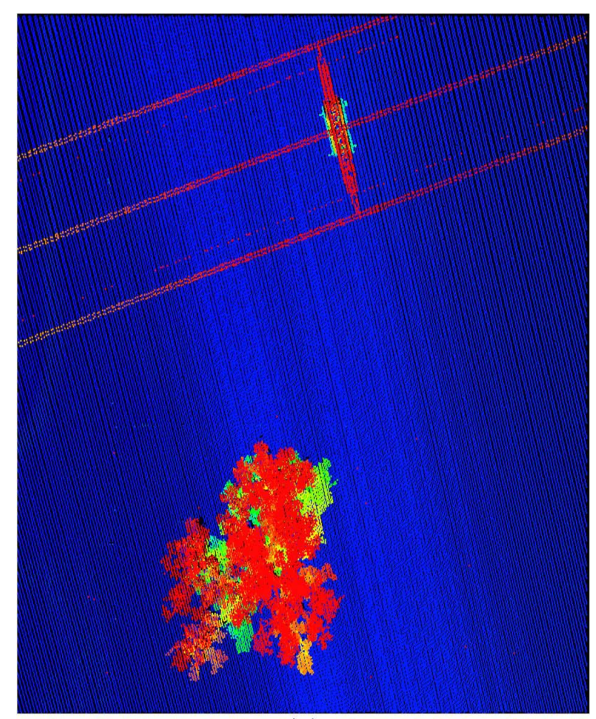

(a)

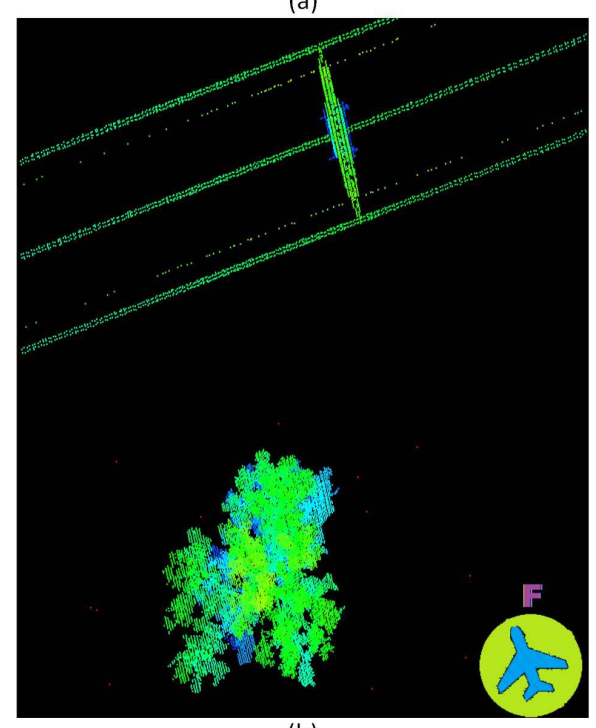

(b)

Fig. 3: Sample scene from Figure 1: (a) all input points and (b) non-ground points. ' $F$ ': flight direction; Point colours: relative heights of points: 'red' = high, 'green' = medium, 'blue' = low.

within a magnified part of 5(a), there are a number of white pixels on each of the wires and so the different components of the power line corridor are not connected. However, after the neighbourhood filling individual components of a power line are found connected in the final mask shown in Figure 5(b). A morphological opening filter [8] with a square structural element $s_{e}$ of size $N$, which is defined in Equation (2) above, is used.

As expected, $M_{m}$ contains pylons and wires connected. Thus, this mask is not suitable for detection of individual pylons. In order to find the pylons, a pylon mask $M_{p}$ is first generated using the same technique as presented above, but only non-ground points below a certain height $T_{H}$ are used. Given the minimum pylon's height is $15 \mathrm{~m}, T_{H}=12 \mathrm{~m}$ is set

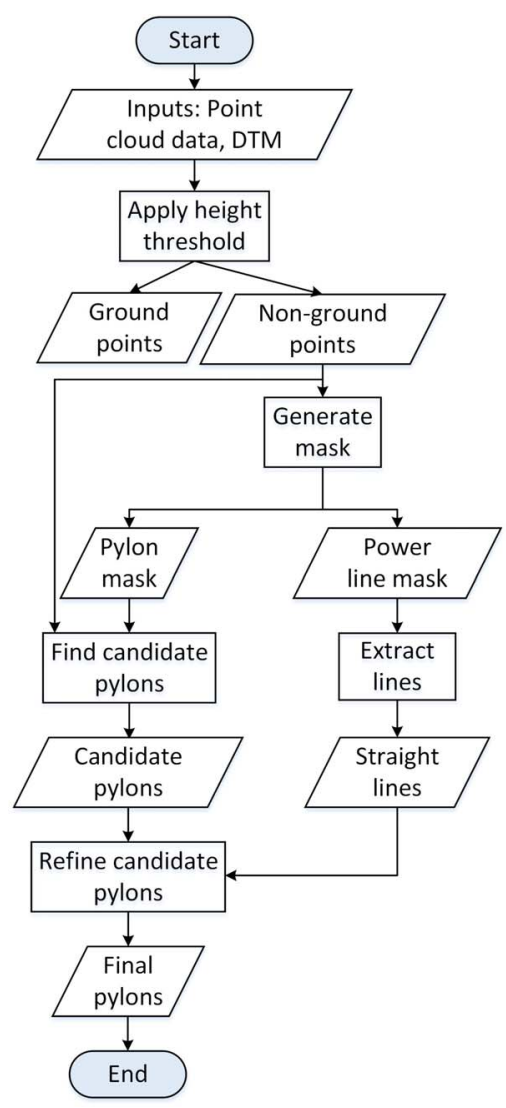

Fig. 4: Flow chart of the proposed pylon detection technique.

in order to avoid the connection between successive pylons. A power line is found in general in the form of a catenary curve and, therefore, its parts connected to the pylons have higher height (close to the pylon) than those away from the pylons. As a consequence, pylons are found stand-alone (without any connected wires) in $M_{p}$.

Since, vertically some pylons may have holes in them, probably due to missing points, $M_{p}$ is then flood filled in order to remove the holes. The MATLAB imfill function [9] is employed for removal of holes. Figure 5(c) shows the pylon mask for the sample scene. As can be observed, this mask contains a true pylon along with the trees in the scene. The single isolated points in the mask are due to noises in the data.

\section{B. Finding Candidate Pylons}

A connected component analysis is then carried out on $M_{p}$ using the bwconncomp function of MATLAB [8]. Components that are larger than a predefined threshold $T_{a}$ are removed as trees. Since, the largest length and width of a pylon are $20 \mathrm{~m}$ and $3 \mathrm{~m}$, respectively, $T_{a}=60 \mathrm{~m}^{2}$ is set in this investigation. Figure 5(d) shows 13 components in different colours. The largest component comprising a number of trees at the bottom of Figure 5(d) is removed at this stage.

Usually, trees and pylons possess different shape properties. For instance, majority of the points on a tree are reflected from branches and leaves, so there may be no or a very small 

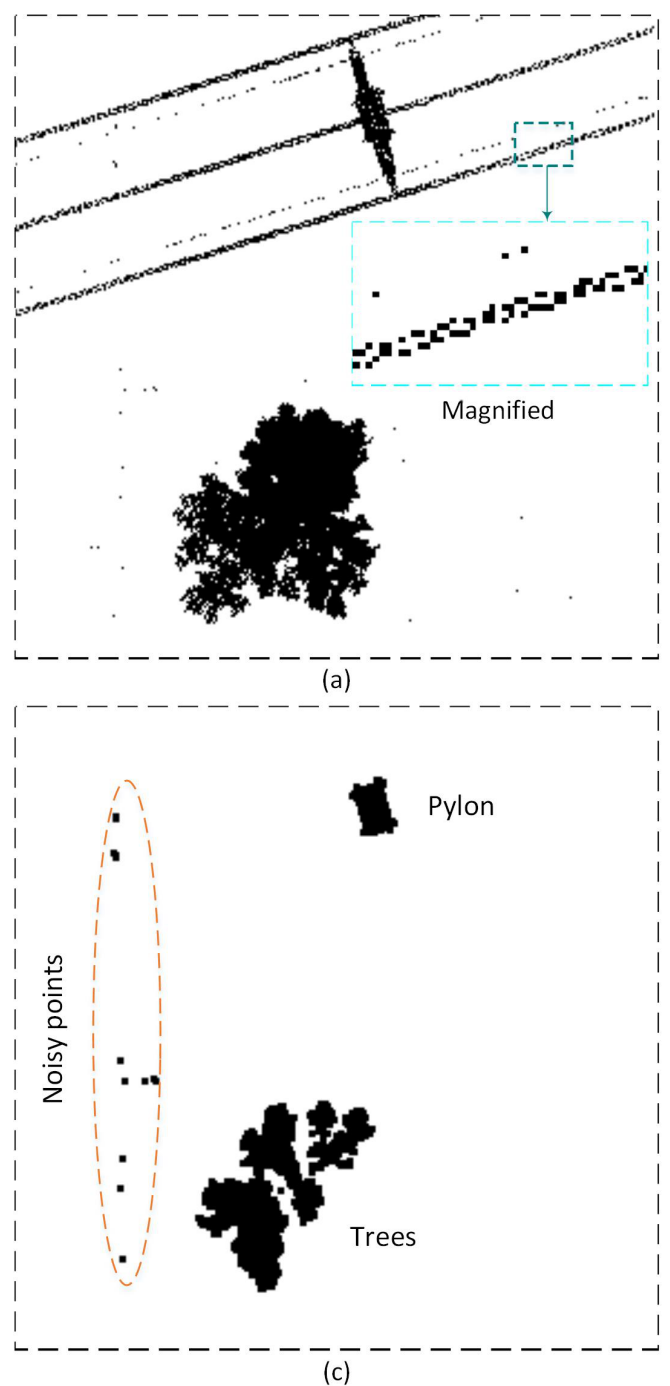

(c)

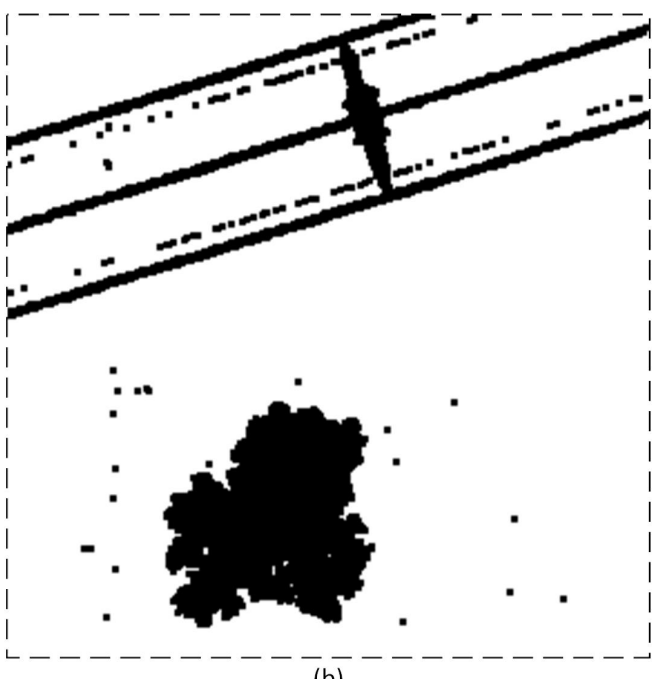

(b)

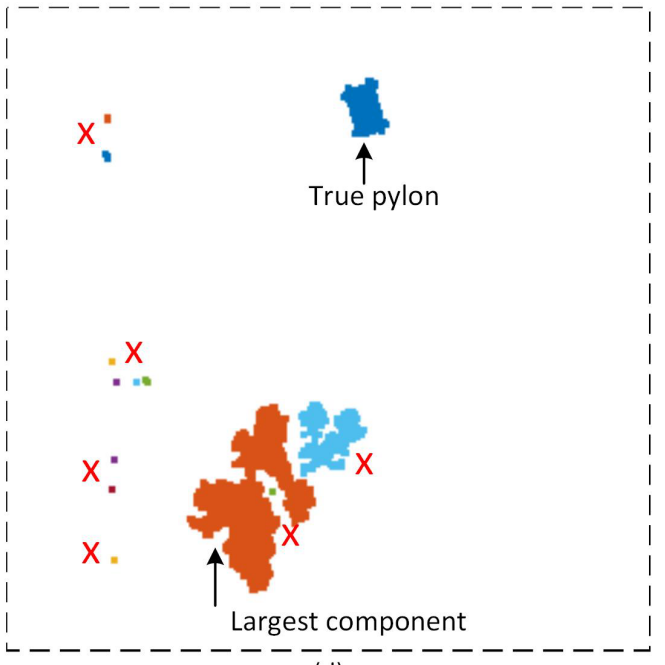

(d)

Fig. 5: Generation of masks: (a) Initial power line mask $M_{m}$,

number of points reaching its trunk. On the other hand, points are reflected from everywhere of a pylon. In addition, pylons can have at least two (horizontal) lines of symmetry, but a tree may not have any such lines of symmetry due to its random shape. Thus, while points on a tree make a asymmetric pattern, those of a pylon make a symmetric pattern.

Based on the above observation, the non-ground points within each of the remaining connected components are analysed as follows to remove the false pylons further. These points are first divided into six histogram bins at $d_{b}=2 \mathrm{~m}$ intervals: 2, 4, 6, 8, 10 and $12 \mathrm{~m}$ (i.e., up to $T_{H}=12 \mathrm{~m}$ ). These height intervals are chosen, since points below $h_{c}=1 \mathrm{~m}$ have been removed as ground points in the mask generation step (see Section III-A) and pylons in the scene are 15 to $30 \mathrm{~m}$ long. Let the histogram of a component be $\left\{b_{i}\right\}$, where $1 \leq i \leq 6$ and $b_{i}$ indicates the number of points in bin $i$. If there are no points at any bins, the corresponding component is straightway removed as a tree. The isolated noisy points also get removed in this stage. For the remaining components, there are points

at all six bins. Due to the symmetric nature of a pylon the number of points in a bin does not differ significantly from other bins. In contrast, for a tree the number of points in a bin should differ significantly from other bins. Figure 6 shows three height histograms for a tree, a pylon and a pole from the test data set in Figure 1(a). For the tree the lower height bins (bin numbers 1 to 4 ) are for the trunk and contain much smaller numbers of points than the higher height bins (bin numbers 5 and 6). As a result, the mean frequency (dashed line) is closer to the low height bins than to the high height bins. In contrast, for both the pylon and the pole the difference in frequencies between two bins is small and the mean frequency line is close to all height bins. Therefore, in order to remove trees and to retain pylons and poles the following two ratios are estimated:

$$
\left\{u_{i}\right\}=\frac{\left\{b_{i}\right\}}{m_{x}}
$$

and

$$
\left\{v_{i}\right\}=\frac{\left\{b_{i}\right\}}{m_{n}}
$$



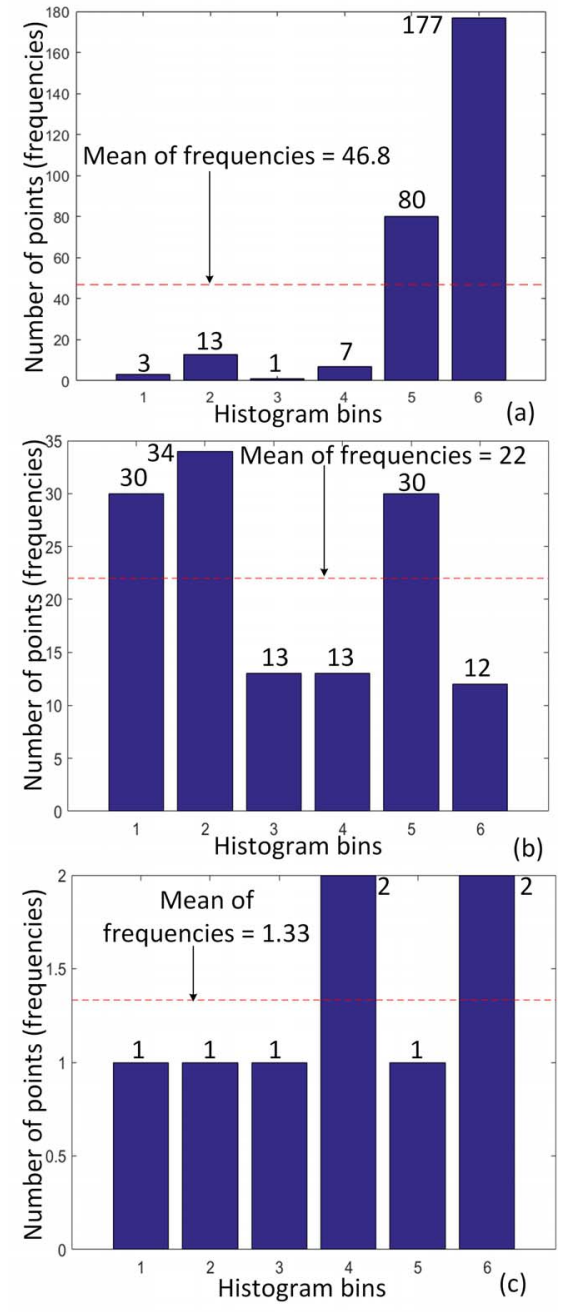

Fig. 6: Height histogram bins: (a) Tree, (b) Pylon and (c) Pole.

where $m_{x}$ and $m_{n}$ are maximum and mean values of $\left\{b_{i}\right\}$. If a connected component has a bin that has a very low number of points $\left(u_{i} \leq T_{u r}=0.1\right)$ or a very high number of points $\left(v_{i} \geq T_{v r}=2\right)$, then that component is removed as a tree. Consequently, the tree (represented in Figure 6(a)) is easily removed although it has 278 points within the considered height range (2 to $12 \mathrm{~m}$ ). The pylon is retained with 132 points from within the height range (Figure 6(b)). Surprisingly, the pole is retained with only 8 points from within the height range (Figure 6(c)). For the sample scene from Figure 3(a), Figure 5(d) shows all 12 removed false pylons with nearby red crosses. The only remaining component is the true pylon in the sample scene.

\section{Finding Final Pylons}

Figure 7(a) shows all the candidate pylons in pink coloured squares after removal of majority of trees using the procedure discussed above. There are still some trees, which exhibit similar characteristics of pylons within the the given height range (2 to $12 \mathrm{~m})$, remain in the mask. In order to remove these trees the following inherent observation is applied.
There are long wires connecting successive pylons in a power line corridor. Nevertheless, such wires do not exist between trees. Therefore, straight lines are first extracted from the power line mask $M_{m}$ and then a constraint of having long straight lines in between nearby candidate pylons is applied.

To extract straight lines from $M_{m}$ the procedure introduced in [10] is followed. Canny edge algorithm is first applied to find edges. Assuming that each power line is at least $l_{m}=6 \mathrm{~m}$ long, edges shorter than $6 \mathrm{~m}$ are removed. Figure 8(a) shows all the surviving edges for the sample scene shown in Figure 3(a).

Corners are then detected on each extracted edge (curve) after smoothing it using a Gaussian convolution filter. The chord-to-point distance accumulation technique is followed to measure curvature at each point of a smoothed curve. Let $\Gamma(t)=(x(t), y(t)), 1 \leq t \leq n_{p}$, be such a smoothed curve and $P_{1}, P_{2}, P_{3}, \ldots P_{n p}$ be the $n_{p}$ points on the curve. To measure the curvature $h_{L}(q)$ at point $P_{q}$ using a chord $C_{L}$ of length $L$, we move the chord on each side of $P_{q}$ at most $L$ points while keeping $P_{q}$ as an interior point. Then, we accumulate all the distances to calculate the discrete curvature at the point $P_{q}$ as

$$
h_{L}(q)=\sum_{j=q-L+1}^{q-1} d_{q, j} .
$$

From the curvature function above non-maxima suppression technique is applied to find curvature extremum points which are corners and thus shows significant direction changes on the curve. Figure 8(a) shows all the smoothed curves and detected corners on them.

A straight line is finally fitted between the two consecutive corners on each extracted edge. Figure 8(c) shows all the extracted lines from the sample scene in cyan colour. Figure 7(b) shows all the extracted lines $\left\{L_{k}\right\}$ in the same colour, where $k \geq 1$, from the test data set.

Thereafter, for each candidate pylon $P_{c}$, other candidate pylons $\left\{P_{o, j}\right\}$, where $j \geq 0$, in its vicinity (considering minimum and maximum distances between successive pylons $108 \mathrm{~m}$ and $450 \mathrm{~m}$, respectively) are obtained. If there are no neighbouring candidate pylons found (i.e., $\left\{P_{o, j}\right\}=\emptyset$ ), $P_{c}$ is removed as an isolated tree. Otherwise, for each $P_{n} \in P_{o, j}$ a line $P_{c} P_{n}$ is generated. All the long lines (at least $l_{m}=6 \mathrm{~m}$ ) from $\left\{L_{k}\right\}$ that are parallel to and reside within the vicinity (i.e., the power line corridor) of $P_{c} P_{n}$ are obtained. If there is no such lines exist between $P_{c}$ and $\forall P_{n} \in P_{o, j}$ then $P_{c}$ is removed as a tree.

Figure 7(b) shows all the pylons that survive after this stage in blue coloured squares and they are considered as the final pylons. The position of each of the pylon is the mean of the non-ground points used to form its connected component in $M_{p}$. The sequence of pylons in each of the three corridors (see Figure 1) can be easily obtained by considering which pylon is a neighbour of which in the above refinement procedure.

\section{PARAmeter Settings}

Table I shows the parameters used by the proposed pylon extraction technique. As can be seen, most of the parameters, 


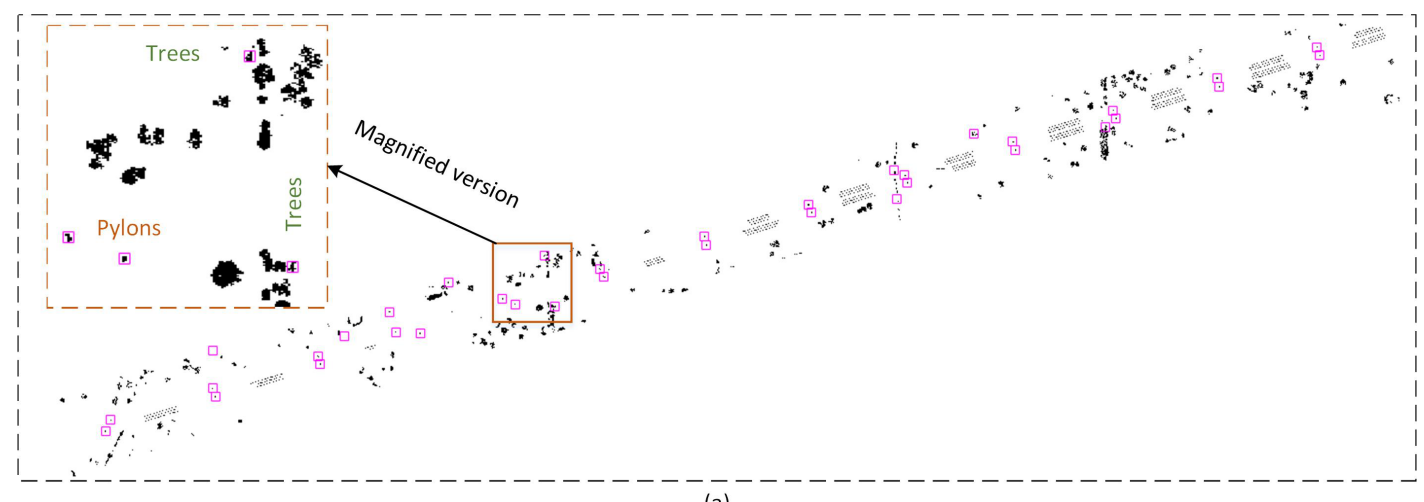

(a)

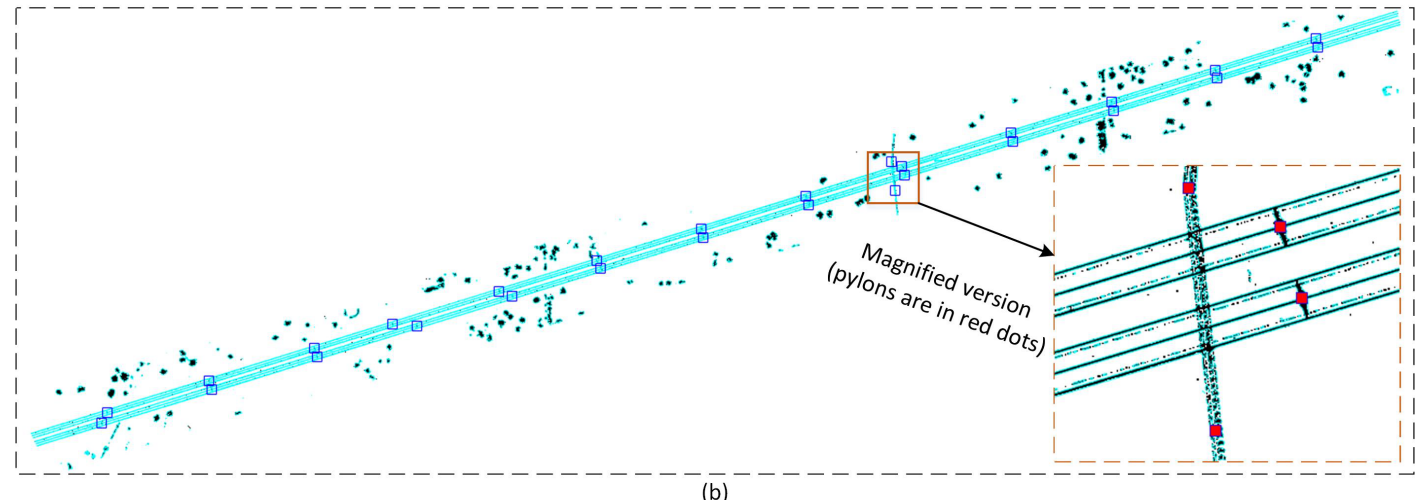

(b)

Fig. 7: Refinement of candidate pylons in the whole test data set: (a) Candidate pylons and (b) Final pylons.

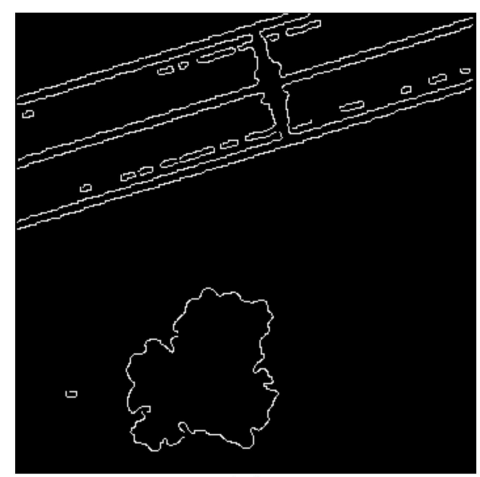

(a)

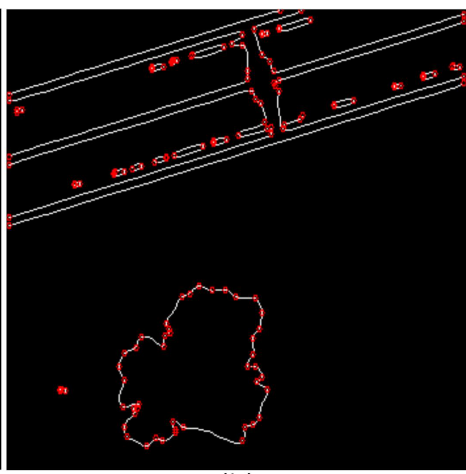

(b)

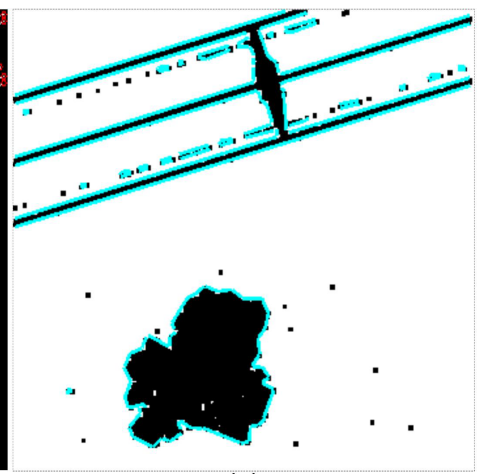

(c)

Fig. 8: Extraction of lines: (a) Canny edges from the power line mask $M_{m}$ from Figure 5, (b) Smoothed edges (white colour) and detected corners (red squares) and (c) Extracted lines using least-square fitting.

such as minimum and maximum distances between pylons and maximum point-to-point distance in point cloud data, are related to the input data and it is assumed that these parameter values are available with the data set. The maximum point height for pylon mask $T_{H}$ is considered $12 \mathrm{~m}$, since the minimum pylon height is $15 \mathrm{~m}$. Many other parameters, such as the resolution of the mask $R_{m}=0.25 \mathrm{~m}$, were previously used for building extraction [11], [12].

The three remaining parameters have been set in this paper. $T_{u r}=0.1$ and $T_{v r}=2$ are used to remove trees. Trees usually have irregular shapes and, therefore, the point density on a tree varies at different heights. At its trunk (low height) region there may be no points or only a few point returns are observed. On the other hand, from its top (high height) parts there may be a large number of point returns. This means that the frequency difference between the smallest and the largest bins is too high. Consequently, each histogram bin for a tree has either too low $\left(u_{i} \leq T_{u r}\right)$ or too high $\left(v_{i} \geq T_{v r}\right)$ number of points. In contrast, a pylon will have points with a regular pattern from top to bottom due to its symmetric shape and thus, the histogram bins have similar number of point returns at all heights. As a result, these thresholds help in effective removal of trees while keep the true pylons.

The minimum power line length is considered as $l_{m}=6$ 
TABLE I: Parameters used by the proposed pylon detection method ('This paper' indicates that a corresponding parameter value has been chosen in this study).

\begin{tabular}{l|l|l}
\hline \hline Parameters & Values & Sources \\
\hline Height constant $h_{c}$ & $1 \mathrm{~m}$ & {$[11]$} \\
Mask resolution $R_{m}$ & $0.25 \mathrm{~m}$ & {$[11]$} \\
Max. point-to-point distance $d_{m}$ & $0.5 \mathrm{~m}$ & Input data \\
Max. point height for pylon mask $T_{H}$ & $12 \mathrm{~m}$ & Input data \\
Area threshold for pylons $T_{a}$ & $60 \mathrm{~m}^{2}$ & Input data \\
Ratio 1 of tree removal $T_{u r}$ & 0.1 & This paper \\
Ratio 2 of tree removal $T_{v r}$ & 2 & This paper \\
Min. distance between pylons & 108 & Input data \\
Max. distance between pylons & 450 & Input data \\
Min. power line length $l_{m}$ & $6 \mathrm{~m}$ & This paper \\
\hline \hline
\end{tabular}

$\mathrm{m}$ and this is far smaller than the length of a real power line between pylons. This threshold is just used to remove too many irrelevant edges and lines which mostly appear from vegetation.

\section{EVALUATION AND COMPARISON}

To our knowledge this research is the second work that detects individual pylons from LIDAR point cloud data. Sohn et al. previously detected pylons as part of their power line scene classification and reconstruction method [5]. They classified the input point cloud data in several object types including pylons. The classification results were presented using the numbers of correctly and incorrectly classified points on pylons. Consequently, an existing performance evaluation result using the number of correctly and incorrectly detected pylons is absent in the state-of-the-art research. Moreover, since the data sets used in the existing research could not be accessed, an appropriate analysis of the comparative results between the proposed and existing techniques is therefore not possible in this investigation.

We evaluate the performance using the object-based completeness and correctness, defined as follows [11]:

$$
\begin{aligned}
C_{m} & =\frac{T P}{T P+F N} \text { and } \\
C_{r} & =\frac{T P}{T P+F P},
\end{aligned}
$$

where $T P$ is the number of truly detected pylons, FP is the number of falsely detected pylons and $F N$ is the number of missing pylons that cannot be detected by the involved pylon detection technique. Since, the proposed technique detected all the true pylons and did not detect any false pylons, both completeness and correctness are $100 \%$. The same performance was obtained by [5] using only 10 pylons. Due to absence of any reference information about the detected pylons, their localisation error cannot be estimated. However, in the magnified version, shown in Figure 7(b), each position is obtained at the centre of the pylon.

\section{CONClusion AND Future WORK}

This paper has presented a new and classifier-free technique for pylon detection from point cloud data. Experimental results show that the proposed technique provides a high pylon detection rate. Since, it does not use any classification algorithms, it avoids the problem associated with classifierbased pylon extraction techniques mentioned in Section I. The height histogram is capable not only to remove many of the trees, but also to detect pylons with only 8 to 10 points reflected from each pylon. The long straight lines representing wires between successive pylons help in elimination of the remaining trees.

In our future investigation, we will extract the individual power line wires through processing the limited number of non-ground points that reside between successive pylons. A parallel computing for achieving a low computation cost is obvious as points in-between a pair of neighbouring pylons can be independently processed. Our future work will also include 3D modelling of power line corridors, specifically each extracted wire will be modelled as a catenary curve [1].

\section{ACKNOWLEDGMENT}

The authors would like to thank the AAM Group (www. aamgroup.com) for providing the test data set. The project is supported by Griffith University's New Researcher Grant (036 Research Internal).

\section{REFERENCES}

[1] J. Ahmad, A. S. Malik, L. Xia, and N. Ashikin, "Vegetation encroachment monitoring for transmission lines right-of-ways: A survey," ISPRS Journal of Photogrammetry and Remote Sensing, vol. 95, no. 2, pp. 339-352, 2013.

[2] B. Guo, Q. Li, X. Huang, and C. Wang, "An improved method for powerline reconstruction from point cloud data," Remote Sensing, vol. 8, no. 1, pp. 1-17, 2016.

[3] L. Matikainen, M. Lehtomäki, E. Ahokas, J. Hyyppä, M. Karjalainen, A. Jaakkola, A. Kukko, and T. Heinonen, "Remote sensing methods for power line corridor surveys," ISPRS Journal of Photogrammetry and Remote Sensing, vol. 19, no. 9, pp. 10-31, 2016.

[4] R. A. McLaughlin, "Extracting transmission lines from airborne LIDAR data," IEEE Geoscience and Remote Sensing Letters, vol. 3, no. 2, pp. 222-226, 2006.

[5] G. Sohn, Y. Jwa, and H. B. Kim, "Automatic powerline scene classification and reconstruction using airborne LIDAR data," ISPRS Annals of the Photogrammetry, Remote Sensing and Spatial Information Sciences, vol. I-3, pp. 167-172, 2012.

[6] H. B. Kim and G. Sohn, "Point-based classification of power line corridor scene using random forests," Photogrammetric Engineering \& Remote Sensing, vol. 79, no. 9, pp. 821-833, 2013.

[7] L. Zhu and J. Hyyppä, "Fully-automated power line extraction from airborne laser scanning point clouds in forest areas," Remote Sensing, vol. 6, no. 11, pp. $11267-11282,2014$.

[8] R. C. Gonzalez, R. E. Woods, and S. L. Eddins, Digital Image Processing Using MATLAB. New Jersey: Prentice Hall, 2003.

[9] P. Soille, Morphological Image Analysis: Principles and Applications. Springer-Verlag Berlin Heidelberg, 1999.

[10] M. Awrangjeb, C. Zhang, and C. S. Fraser, "Building detection in complex scenes thorough effective separation of buildings from trees," Photogrammetric Engineering \& Remote Sensing, vol. 78, no. 7, pp. 729-745, 2012.

[11] M. Awrangjeb and C. S. Fraser, "Automatic segmentation of raw LIDAR data for extraction of building roofs," Remote Sensing, vol. 6, no. 5, pp. 3716-3751, 2014.

[12] S. A. N. Gilani, M. Awrangjeb, and G. Lu, "Robust building roof segmentation using airborne point cloud data," in IEEE International Conference on Image Processing, Phoenix, Arizona, USA, 2016, pp. 859-863. 